\title{
Isolated Unilateral Hypoglossal Nerve Palsy Due to Vertebral Artery Dissection
}

\author{
Karthik Mahadevappa, MBBS; Thomas Chacko, MD; and Anil K. Nair, MD
}

\begin{abstract}
We report the case of a patient with unilateral tongue weakness secondary to an isolated lower motor neuron hypoglossal nerve palsy that was caused by a right vertebral artery dissection in the lower neck. The patient had a boggy tongue with a deviation to the right side but an otherwise normal neurological examination. Magnetic resonance angiography showed a narrow lumen of the right vertebral artery in the neck. After initially treating the patient with aspirin in the emergency room and later with warfarin for three months, there was complete recanalization of the right vertebral artery. Only one other case of vertebral artery dissection and twelfth nerve palsy has been reported before.
\end{abstract}

Keywords: Dysarthria; Hypoglossal nerve; Lateral medullary syndrome; Tongue weakness; Vertebral artery dissection

Corresponding Author:

Anil K. Nair, MD

Chief of Neurology, Quincy Medical Center

Director, Alzheimer's Disease Center

I 4 Whitwell Street, 4th floor

Quincy, MA 02169, USA

Tel: (6I7) 302-6388

G.Voice: (617) 639-5006

Fax: (6I7) 934-2425

Email: nair.anil@gmail.com

Received: June 16, 2011

Revised: August II, 2011

Accepted: August 17, 20II

doi: $10.3121 / \mathrm{cmr} .2011 .1029$ he hypoglossal nerve (CN XII) is the motor nerve of the tongue. Isolated hypoglossal nerve paralysis is uncommon. Vascular, inflammatory, traumatic, or space-occupying lesions can affect the CN XII anywhere in its course, from the nucleus in the caudal medulla, through the intracranial extramedullary portion of the nerve, across the hypoglossal canal, or into its extracranial termination. ${ }^{1-4}$ The rootlets cross the premedullary cistern superior to the vertebral artery and exit the skull base through the hypoglossal canal. ${ }^{1,3}$ This close relationship can cause compression of rootlets intracranially when there is vertebrobasilar pathology or anatomic variants of the vertebral artery resulting in paralysis of the nerve. ${ }^{3-5}$ The rootlets join to form one or two trunks before piercing through the dura. ${ }^{1,2}$ The nerve then descends almost vertically to a point corresponding with the angle of the mandible. In the neck it is deeply seated beneath the internal carotid artery, internal jugular vein, and vagus nerve. Lower in the neck the nerve emerges superficially just below the digastric muscle. ${ }^{2}$

\section{Case Report}

A woman, 20 years old, presented to the emergency room with sudden onset of slurring of speech, lasting for about an hour. This was preceded by a two week period of neck pain. The patient took acetaminophen and ibuprofen with limited relief. There was no history of trauma, nausea, vomiting, headache, blurring of vision, double vision, or dysphagia; however, she felt dizzy five seconds before the symptoms appeared. On examination, the patient was normotensive, dysarthric, and afebrile. She scored a 2 on the NIH stroke scale. The dysarthria was due to difficulty in tongue movement. She could not repeat "Methodist Episcopal," "Huckleberry Finn," and "Fifty-Fifty," but she was able to repeat 


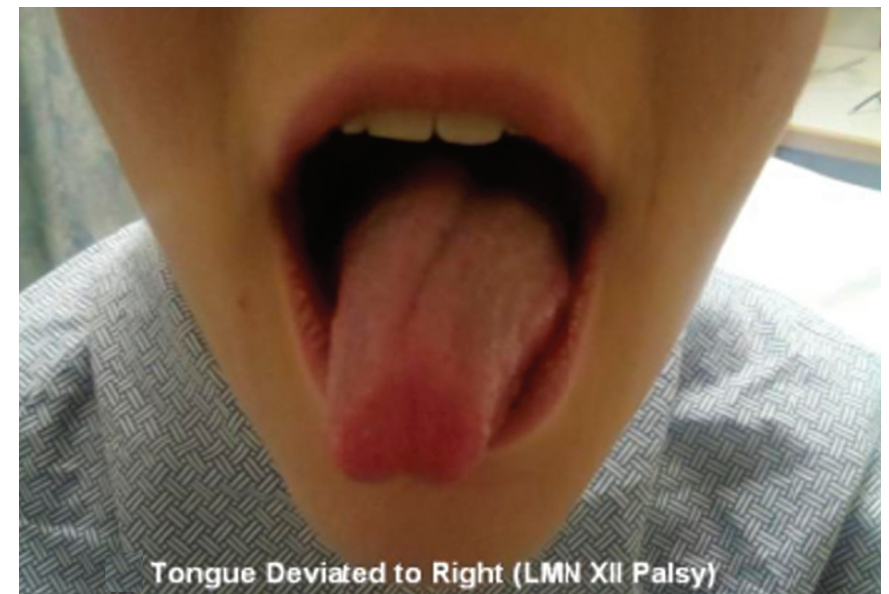

Figure 1. Patient presented with a boggy tongue deviated to right side.

"Mama" and "Tip-Top". She had a boggy tongue that was enlarged on the left side which led to difficulty in movement. Her tongue deviated to the right side on protrusion. With consent, a picture of the tongue movement was obtained (figure 1). The remaining cranial nerve examination as well as the rest of her neurological examination was unremarkable.

A laboratory evaluation with complete blood count and electrolyte assessment was normal. Antithrombin III and lupus anticoagulant were normal, while protein $\mathrm{C}$ was lower than expected. A computed tomography (CT) scan of the head showed no intracranial abnormality. The patient was treated with aspirin initially in the emergency room and was transitioned to warfarin for three months. Diffusion-weighted magnetic resonance imaging of the brain was normal (figure 2A), but magnetic resonance angiography of the neck showed narrowing of the right vertebral artery. The vertebral artery narrowed approximately $2 \mathrm{~cm}$ from its origin until the level of $\mathrm{C} 2$, suggesting vertebral dissection (figure $2 \mathrm{~B}$ and 2C). A CT angiogram of the neck confirmed right vertebral artery dissection exhibiting a double lumen, with the true lumen narrowed throughout its course, as suggested above (figure 2D). Both bilateral carotids and the left vertebral artery were normal.

The patient's hypoglossal nerve palsy weakness improved with aspirin and warfarin, and there was no recurrence of her symptoms in the aftermath of the dissection. No tongue atrophy was seen at one month (figure $3 \mathrm{~A}$ ) or at her three month follow-up appointments (figure 3B). Also at 3 months, imaging of the right vertebral artery showed complete recanalization (figure 3C). Our patient did not have lateral medullary syndrome, as there was no vertigo. Imaging showed that the lateral portion of the medulla oblongata was normal (figure 4A and 4B). She had no sensory deficits affecting the face or extremities on the contralateral side, no dysphagia or pain on the ipsilateral side of the face, and her corneal reflex was intact. There was no Horner syndrome or loss of pain and temperature sensation.
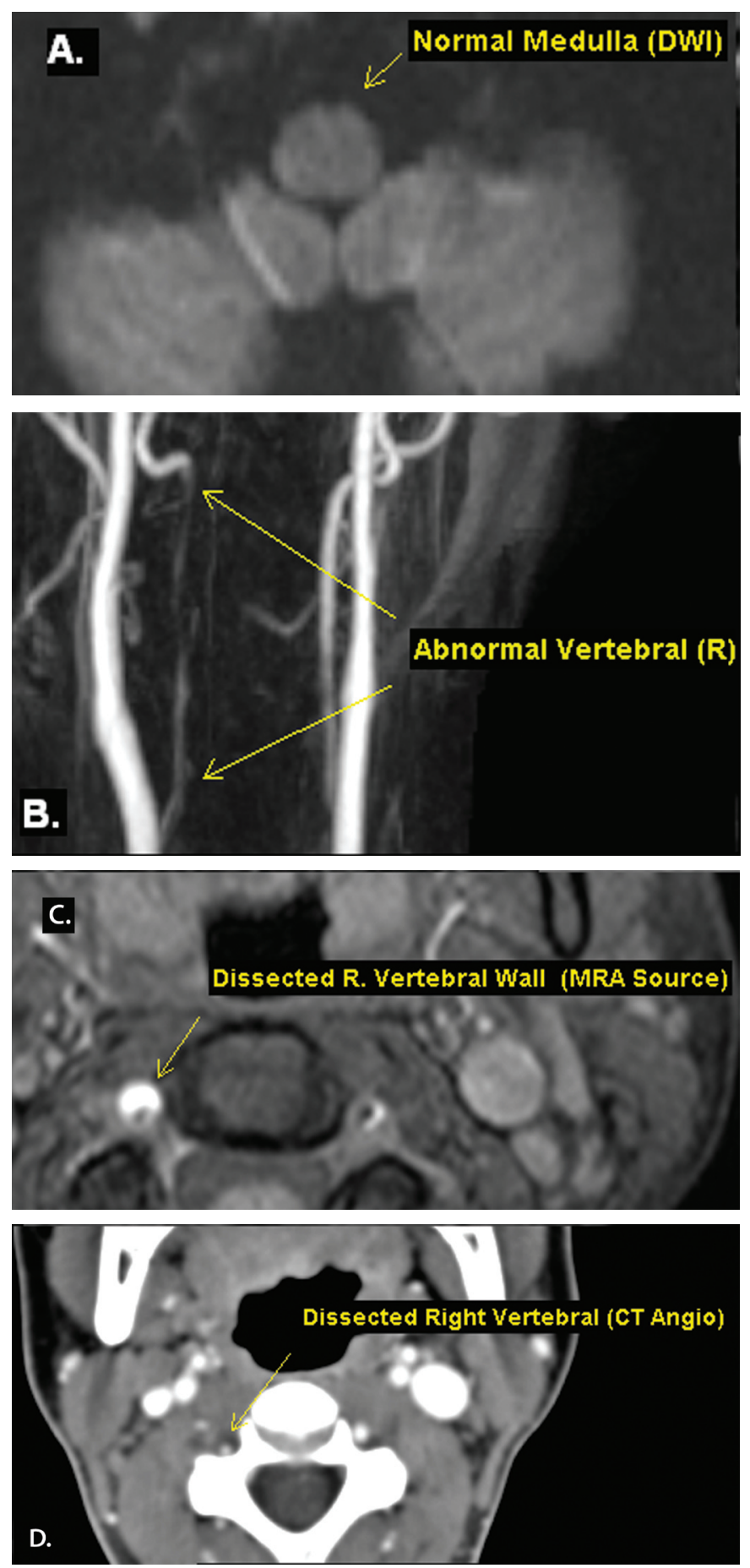

Figure 2. (A) Diffusion-weighted magnetic resonance image (DWI) showing normal medulla demonstrates normal signal, without evidence of infarct. (B) T1-weighted fat-saturated image demonstrating cresentric high signal within the right transverse foramen, consistent with intramural hematoma within the right vertebral artery. (C) Contrast-enhanced magnetic resonanceangiography (MRA) unambiguously demonstrating a double lumen effect in the extracranial portion of right vertebral artery, consistent with a dissection. (D) Contrast-enhanced computed tomography angiogram (CT angio) showing a double lumen, with the true lumen narrowed, indicating a dissection of right vertebral artery. 

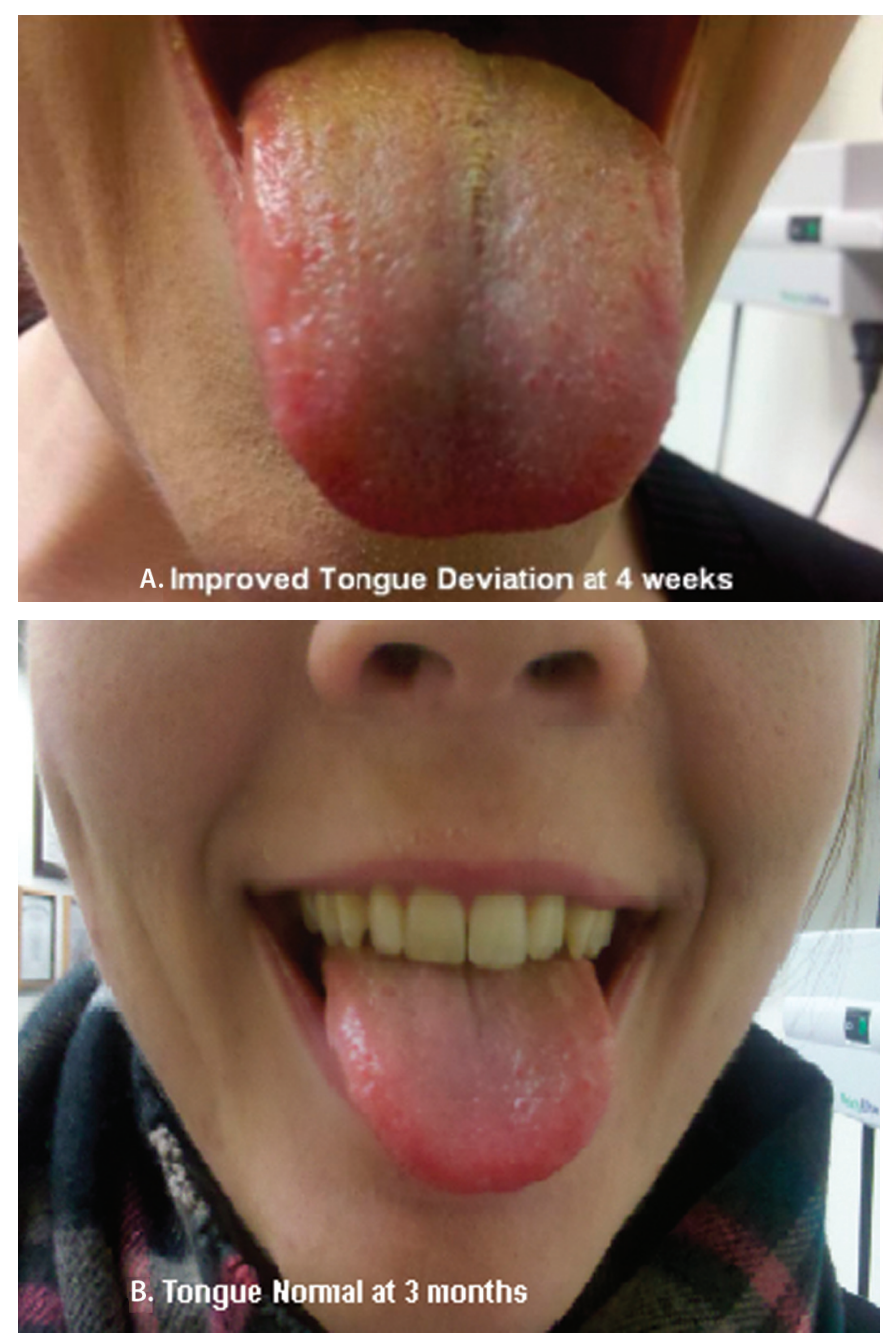

Figure 3. One month after treatment, the patient showed (A) no tongue atrophy. Three months after treatment the patient had (B) a normal tongue, and (C) complete recanalization of the right vertebral artery.

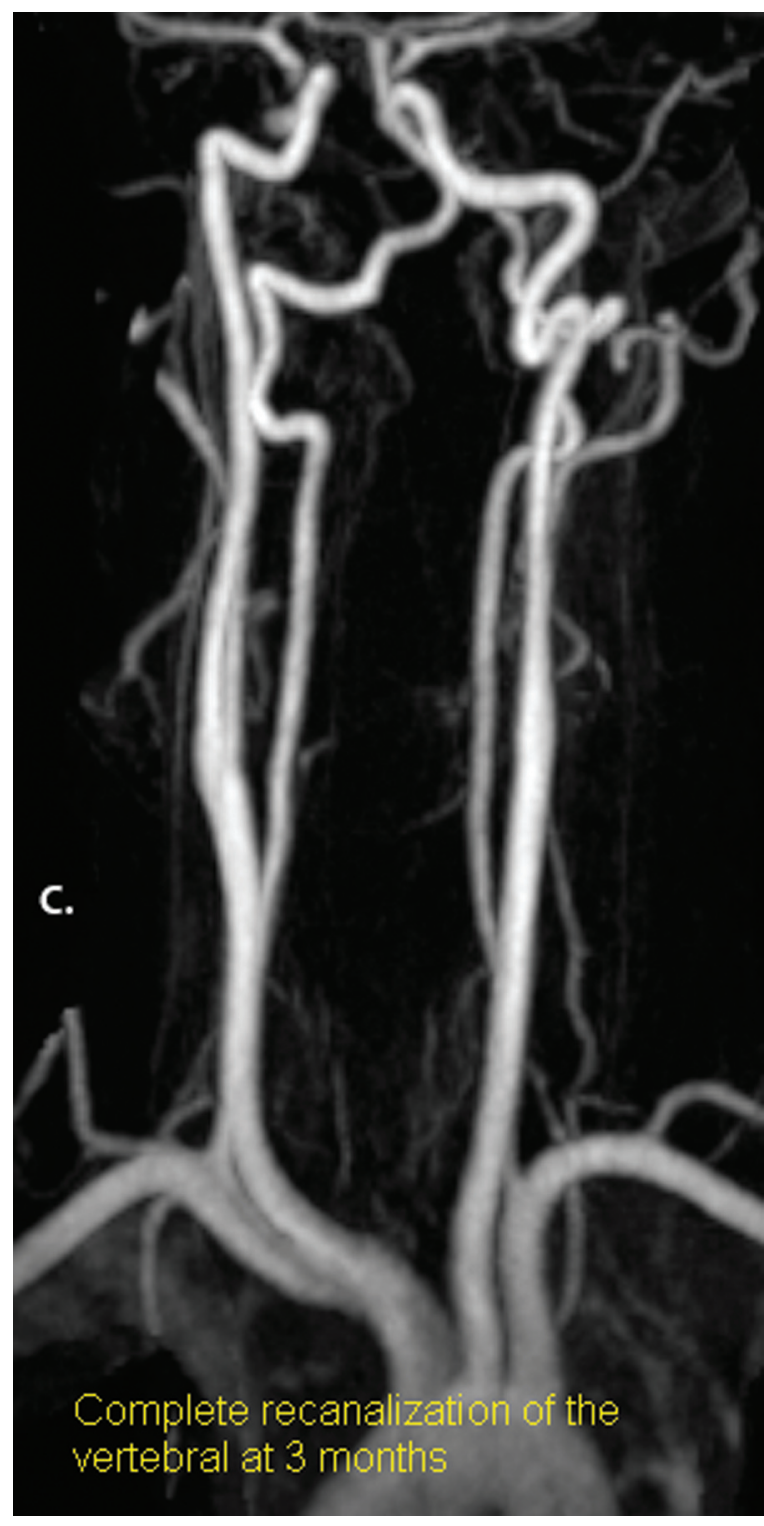

\section{Discussion}

This case is unique, as unilateral isolated CN XII palsy is uncommon when vertebral artery dissection occurs outside the premedullary cistern. Upper motor neuron and nuclear lesions are usually bilateral due to the close proximity of $\mathrm{CN}$ XII nuclei. In our patient there was no evidence of extension of the extracranial dissection into the intracranial vasculature; therefore, direct compression of CN XII rootlets was unlikely. There was no subarachnoid blood on the CT scan, and our patient did not want to have a lumbar puncture. The more plausible cause for CN XII infarction was distal embolization from the extracranial vertebral artery to the vasa nervorum of the distal intracranial arteries.

CN XII is closely related to both the carotid and vertebral arteries, and it has been described in the setting of various vascular pathologies. ${ }^{3,46}$ The proximal cisternal portion of CN XII is mainly supplied by vertebral branches. ${ }^{4,6,7}$ CN XII receives its distal blood supply predominantly from the carotid circulation via the ascending pharyngeal artery, the occipital artery, the external carotid artery, and the lingual artery. ${ }^{4,6}$

Most cases of vertebral artery dissection are thought to be spontaneous events. However, dissection can be precipitated by minor trauma or by spinal manipulation. Connective tissue disorders such as fibromuscular dysplasia, Marfan's syndrome, and Ehlers-Danlos syndrome type IV increase the risk for cervical arterial dissection. ${ }^{4}$ Vertebral artery dissection is an important cause of posterior circulation ischemia, particularly in young and middle-aged patients. ${ }^{4}$ Features of lateral medullary syndrome, neck pain, vertigo, nausea, and vomiting are common. ${ }^{4,8,9}$ None of these were present in our subject. Treatment with warfarin may be more effective than aspirin in preventing recurrence of ischemic symptoms. ${ }^{4,10}$

\section{Conclusion}

We describe an unusual case of isolated unilateral hypoglossal nerve palsy due to vertebral artery dissection in the lower 

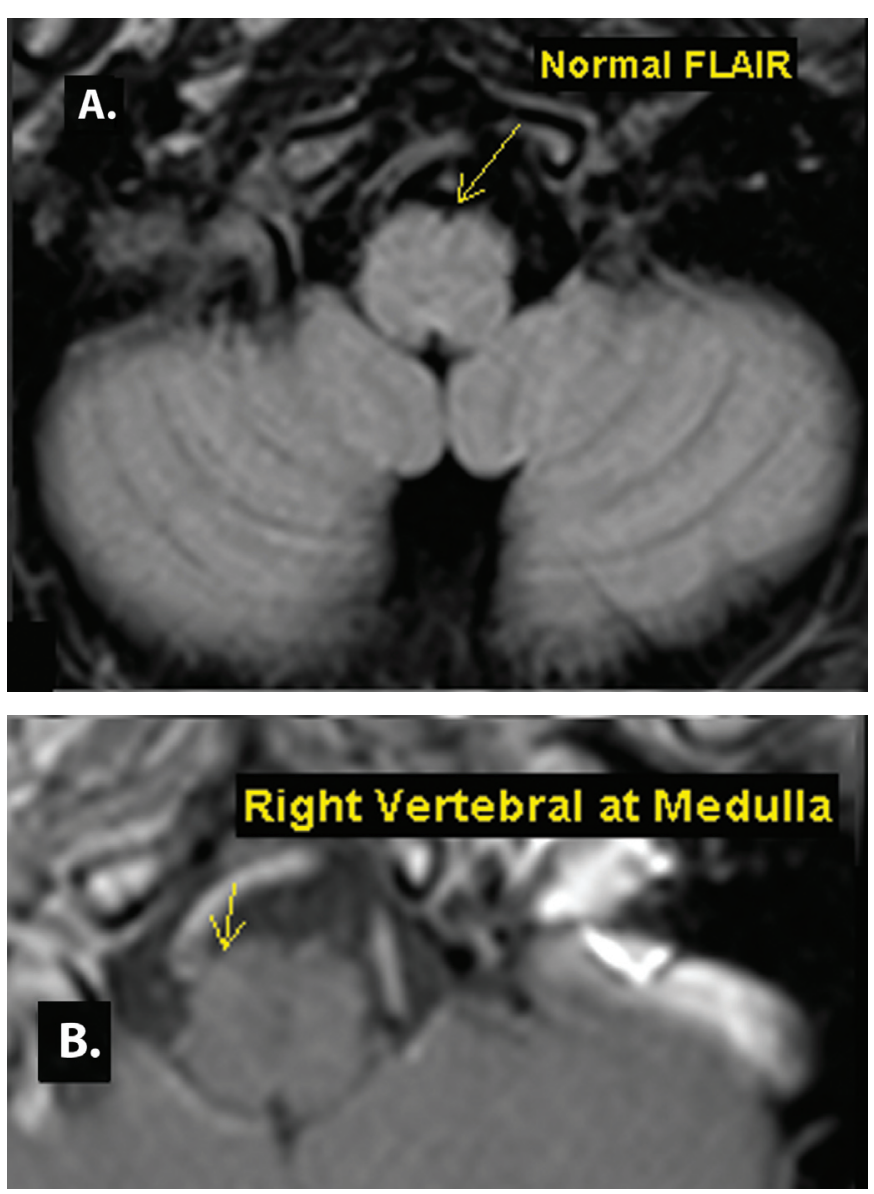

Figure 4. (A) FLAIR image showing normal medulla with no signal abnormalities. (B) T1 image of the medulla showing right vertebral artery.

neck, which has been reported only once before. In our case, clinical and neuroimaging evidence led us to conclude that embolization from the extracranial vertebral artery to the vaso nervorum of the distal intracranial arteries resulted in the subjects CN XII infarction.

We would like to emphasize the following learning points:

- This case highlights the need for complete neurological examination in young patients and some knowledge of differential diagnosis.

- In a young patient with cranial nerve palsy, the possibility of stroke should always be considered.

- Vertebral branches mainly supply the proximal cisternal portion of the hypoglossal nerve.

- Diagnosis of dissection of vertebrobasilar artery may reduce complications of posterior fossa infarction and subarachnoid hemorrhage.

\section{References}

1. Loh C, Maya MM, Go JL. Cranial nerve XII: the hypoglossal nerve. Semin Ultrasound CT MR 2002; 23:256-265.

2. Henry G. The Hypoglossal Nerve. Human Anatomy: Gray's Anatomy Website. Available at: http://www.theodora.com/ anatomy/the hypoglossal nerve.html. Accessed April 4, 2011.
3. Morini A, Rozza L, Manera V, Buganza M, Tranquillini E, Orrico D. Isolated hypoglossal nerve palsy due to an anomalous vertebral artery course: report of two cases. Ital J Neurol Sci 1998; 19:379-382.

4. McKeon A, Murphy S, McNamara B, Ryder DQ, Galvin RJ. Isolated hypoglossal nerve palsy due to compression by a dissecting vertebral artery. Eur Neurol 2005;53:162-164.

5. Alves P. Imaging the hypoglossal nerve. Eur J Radiol 2010; 74:368-377.

6. Bademci G, Yaşargil MG. Microsurgical anatomy of the hypoglossal nerve. J Clin Neurosci 2006; 13:841-847.

7. Gibo H, Marinković S, Nikodijević I, Stimec B, Erden A. The blood supply of the hypoglossal nerve: the microsurgical anatomy of its cisternal segment. Surg Neurol 1997; 48:85-91.

8. Bonkowsky V, Steinbach S, Arnold W. Vertigo and cranial nerve palsy caused by different forms of spontaneous dissections of internal and vertebral arteries. Eur Arch Otorhinolaryngol 2002; 259:365-368.

9. Kim JS. Pure lateral medullary infarction: clinical-radiological correlation of 130 acute, consecutive patients. Brain 2003; 126:1864-1872.

10. Kim YK, Schulman S. Cervical artery dissection: pathology, epidemiology and management. Thromb Res 2009; 123:810-821.

\section{Author Affiliations}

Karthik Mahadevappa, MBBS*; Thomas Chacko, $M D^{+}$; and Anil K. Nair, MD*

*Department of Neurology, Quincy Medical Center/ Alzheimer's Disease Center, Quincy, Massachusetts, USA Department of Radiology, Quincy Medical Center, Quincy, Massachusetts, USA 